\title{
BRYOFLORISTICAL DATA FROM THE APUSENI MOUNTAINS (ROMANIAN WESTERN CARPATHIANS, TRANSILVANIA) 2.
}

\author{
Andrea Sass-Gyarmati* \& Tamás Pócs \\ Eszterházy Károly University, Institute of Biology, Department of Botany and Plant \\ Physiology; Eger, Pf. 43, H-3301 Hungary; *E-mail: sassgyarmati@gmail.com

\begin{abstract}
The main aim of this study was to explore the bryophyte diversity and distribution patterns in the Apuseni Mountains. From our collections hitherto 94 bryophyte species were identified. The 25 Marchantiophyta and 69 Bryophyta species belong to 73 genera of 42 families. Syntrichia norvegica are new for the whole Apuseni Mountains. Among them the endangered Campylium protensum, the near threatened Barbula crocea, Platydictya jungermannioides and the very rare Abietinella abietina var. hystricosa are worth to be mentioned.
\end{abstract}

Keywords: bryoflora, rare species, Apuseni Mts., Romania

Rezumat: Lucrarea prezintă distribuția speciilor de briofite din cadrul Munților Apuseni. Din colecţia recentă fost identificate 94 de specii de briofite. Cele 25 specii de Marchantiophyta și 69 de specii de Bryophyta aparțin in 73 de genuri si 42 de familii. Syntrichia norvegica este semnalare nouă pentru Munţii Apuseni. Campylium protensum este specie periclitată, Barbula crocea şi Platydictya jungermannioides specii amenințate iar Abietinella abietina var. hystricosa specie rară care merită să fie menționată.

Cuvinte cheie: brioflora, specii rare, Apuseni, România

\section{INTRODUCTION}

The first bryological records of the climatologically and geologically very variable and biologically very rich Apuseni Mountains were published at the end of XIX th century (Csató 1885), but its intensive investigation started only in the $\mathrm{XX}^{\text {th }}$ century, which till now is far from complete (Győrffy 1903; Péterfi 1908, 1910; Boros 1942a, 1942b, 1951; Páll 1960, 1962, 1963; Raţiu et al. 1966; Boros and Vajda 1967, 1974; Ştefureac 1975, 1977; Plămadă and Goia 1994). Investigations have continued intensively in the past twenty years 
(Goia 2001, 2005; Goia and Mătase 2001; Jakab 1999, 2000; Goia and Schumacker 2000, 2002, 2003a, 2003b, 2004; Goia and Ştefănuţ 2004; Plămadă et al. 2000; Lüth 2002, and others). Csürös (1981) gave a wide overview of the natural conditions and of Apuseni Mountains and its flora and fauna. A preliminary report on the Bihor mountains was published by Simon (1960) and a very detailed vegetation study is given by Pop et al. (2000) from its part in Cluj County. We started our bryological exploration in 2002 and published a series of papers from this area, some of them as result of the fruitful cooperation with Irina Goia, bryologist at the BabeşBolyai University (Pócs 2005, Sass-Gyarmati et al. 2005a, 2005b, Sass-Gyarmati et al. 2008a, 2008b, Sass-Gyarmati and Pócs 2017).

\section{Study area}

Muntele Mare and Gilăului Mountains form a distinct geomorphological unit, known as Gilău - Muntele Mare. The massif is covered with beech and spruce forests. The crystalline schists gave rise to a massive landscape, with wide interfluves separated by narrow and deep valleys. Reserve includes "Şesul Craiului" located on a limestone plateau above $1350 \mathrm{~m}$ altitude, the forest on the mountain scale and spectacular cliffs guard Belioara Valley (tributary of Poşaga Valley), with vertical walls, tanks, gully and sharp increases. It has an area of 47.7 hectares and houses many rare species of plants and animals. Initiative put under protection of the area had Alexandru Borza, since the early 20's of the twentieth century. Here have found their place those floral rarities and a relic forest of conifers (scotch pine and larch) for which in 1935 it was declared a botanical reservation. Csürös (1958) presents in details vegetational studies made in Scărişoara-Belioara (Bélavár) Massif and published several more studies, partly together with CsürösKáptalan.

The Bihor Mountains occupy a central position within the Apuseni mountains range. The characteristic karstic topography is widely developed, parallel to places with other sedimentary and volcanic bedrocks. The Bihar Massif is the highest part of the Apuseni Mountains. Here the general aspects of vegetation of Cheile Ordâncuşa - a narrow, $2.5 \mathrm{~km}$ long limestone gorge - were studied by I. Pop and I. Hodişan (1967). The streamlet has the side-branch of Gârdei Seacă on the left part. The Ordâncușa gorge has $400 \mathrm{~m}$ high limestone walls, between which there is a road leading to the 
Scărişoara Ice Cave. In the cliffs more than 70 caves, gates and smaller halls are present. Among them the most popular is Poarta lui Ionele cave with different species of protected bats. In the right wall can be found the Zgurăști sinkhole, with interesting vegetation inversion and with an underground lake (Sass-Gyarmati et al. 2005a). The characteristics of the landscape are crevices, extended karst zones (with almost 430 caves described) and the third largest underground ice cave of Europe after the Eisriesenwelt cave in Austria and Dobsinska cave in Slovakia (Strug et al. 2006), the "Ghețarul de la Scărişoara" - which is the largest in Romania, declared a natural monument and a speleological reserve. It is situated at $1165 \mathrm{~m}$ a.s.l., the entrance being located on the western wall of a circular shaft $60 \mathrm{~m}$ in diameter and $47 \mathrm{~m}$ deep. Beyond the entrance, the ice block with a volume of $100000 \mathrm{~m}^{3}$ and area extent of $3000 \mathrm{~m}^{2}$ (Perşoiu et al. 2011).

\section{MATERIAL AND METHODS}

The byophytes enumerated below were collected from Apuseni Mountains between 22-25 October 2006 by Tamás Pócs, Irina Goia, Zoltán Tóth and identified by Tamás Pócs and Andrea SassGyarmati. The collection was made in various vegetation types: bogs, meadows, beech and spruce forests and subalpine belts. The Romanian distribution of mosses was established from Plămadă (1998) and Mohan (1998), while that of the liverworts from Ştefănuţ (2008). The nomenclature of liverworts follows Ştefănuţ (2008) modified by Söderström et al. (2016), nomenclature of mosses follows Hill et al. (2006), except Racomitrium affine which was recently included to Bucklandiella (F. Weber \& D. Mohr) Bednarek-Ochyra \& Ochyra (Ochyra et al. 2003). The classification of liverworts (Marchantiophyta) follows Söderström et al. (2016), while the classification of mosses (Bryophyta) follows Goffinet and Shaw (2009). The species in each family are arranged in alphabetical order. Species names are followed by the collecting site number, and by the substrate on which they were grown. The collected specimens are deposited in the Herbarium of Eger (EGR). The collecting sites are listed in the Appendix. 


\section{RESULTS}

\section{List of species}

During the field study, 94 bryophyte species were found in the investigated area. The 25 Marchantiophyta and 69 Bryophyta species belong to 73 genera of 42 families.

\section{Marchantiophyta}

Conocephalaceae

Conocephalum conicum (L.) Dumort. - 13: on irrigated rocks

Marchantiaceae

Preissia quadrata (Scop.) Nees - 2: on limestone rocks

Aneuraceae

Aneura pinguis (L.) Dumort. - 2, 12: on limestone rocks

Anastrophyllaceae

Barbilophozia barbata (Schmidel ex Schreb.) Loeske - 9: on volcanic rocks

Lepidoziaceae

Bazzania trilobata (L.) Gray. - 5: lignicolous

Lepidozia reptans (L.) Dumort - 5: on volcanic rocks; 10: lignicolous

$$
\text { Cephaloziaceae }
$$

Cephalozia pleniceps (Austin) Lindb. - 13: on vertical cliff

Lophoziaceae

Lophozia ventricosa (Dicks.) Dumort. - 5: on volcanic rocks.

\section{Scapaniaceae}

Scapania aequiloba (Schwagr.) Dumort. - 12: on vertical cliff

Scapania calcicola (S.W. Arnell \& J. Perss.) Ingham - 13: on limestone rocks

Scapania nemorea (L.) Grolle - 5, 9: on volcanic rocks

Jubulaceae

Frullania dilatata (L.) Dumort. - 1, 3, 12, 13: on bark 


\section{Jungermanniaceae}

Jungermannia atrovirens Dumort. - 12, 13: on limestone rocks Tritomaria exsecta (Schmidel) Schiffn. ex Loeske - 5, 10: lignicolous

\section{Blepharostomataceae}

Blepharostoma trichophyllum (L.) Dumort - 5: on volcanic rocks; 10: lignicolous

Lophocoleaceae

Lophocolea heterophylla (Schrad.) Dumort. - 8, 12: on decaying $\log$

Plagiochilaceae

Pedinophyllum interruptum (Nees) Kaal. - 12: on limestone rocks Plagiochila porelloides (Torr. ex Nees) Lindenb. - 12: on limestone rocks

Trichocoleaceae

Trichocolea tomentella (Ehrh.) Dumort. - 5: twigs of a dead spruce

$$
\text { Radulaceae }
$$

Radula complanata (L.) Dumort. - 12, 13: on bark

Ptilidiaceae

Ptilidium pucherrimum (Weber) Hampe - 10: on spruce roots

$$
\text { Metzgeriaceae }
$$

Apometzgeria pubescens (Schrank) Kuwah. - 12: on limestone rocks

Metzgeria conjugata Lindb. - 12: on bark

\section{Lejeuneaceae}

Cololejeunea calcarea (Lib.) Schiffn. - 13: on limestone rocks Lejeunea cavifolia (Ehrh.) Lindb. - 13: on limestone rocks

\section{Bryophyta}

\section{Sphagnaceae}

Sphagnum angustifolium (C.E.O. Jensen ex Russow) C.E.O. Jensen 7: transition spring bog

Sphagnum capillifolium (Ehrh.) Hedw. - 5, 8: on raised bog Sphagnum fuscum (Schimp.) Klinggr. - 8: on raised bog Sphagnum girgensohnii Russ. -5 , 8: on turf 
Sphagnum magellanicum Brid. - 6, 8: on turf

Sphagum subsecundum Nees - 7: transition spring bog

Tetraphidaceae

Tetraphis pellucida Hedw. - 10: lignicolous

Polytrichaceae

Polytrichum strictum Menzies - 8: on raised bog

Polytrichum longisetum Sw. ex Brid. - 5: on peaty soil

Encalyptaceae

Encalypta streptocarpa Hedw. - 1: on limestone rocks

Encalypta vulgaris Hedw. - 1: on limestone rocks

\section{Grimmiaceae}

Grimmia hartmanii Schimp. - 9: on volcanic rocks.

Bucklandiella affinis (F. Weber \& D. Mohr) Bednarek-Ochyra \& Ochyra, Syn.: Racomitrium affine (F. Web. et D. Mohr) Lindb. - 5: on soil

Schistidium apocarpum (Hedw.) Bruch \& Schimp. - 10: on limestone rocks

$$
\text { Seligeriaceae }
$$

Seligeria acutifolia Lindb. - 13: on vertical cliff

Seligeria patula (Lindb.) I. Hagen - 13: on vertical cliff

\section{Fissidentaceae}

Fissidens adianthoides Hedw. - 12: on limestone rocks

Fissidens dubius P. Beauv. - 2: on limestone rocks

\section{Dicranaceae}

Dicranella heteromalla (Hedw.) Schimp. - 10: lignicolous

Dicranum scoparium Hedw. - 5: on bog; 10 on Picea roots; 11: on rotten spruce stumps

Dicranum fuscescens Sm. - 8: on raised bog

Dicranum montanum Hedw. - 10: on rotten spruce stumps

Paraleucobryum longifolium (Ehrh. ex Hedw.) Loeske - 5: on volcanic rocks

\section{Pottiaceae}

Barbula crocea (Brid.) F. Weber \& D. Mohr - 2, 13: on limestone rocks 
Bryoerythrophyllum recurvirostrum (Hedw.) P.C.Chen - 9: on volcanic rocks

Didymodon fallax (Hedw.) R. H. Zander - 2: on vertical cliff

Didymodon ferrugineus (Schimp. ex Besch.) M.O.Hill - 2: on limestone rocks

Gymnostomum aeruginosum Smith. - 13: on vertical cliff

Syntrichia norvegica F. Weber - 10: on limestone rocks

Tortella tortuosa (Hedw.) Limpr. - 1, 2: on soil; 10, 12: on limestone rocks

$$
\text { Orthotrichaceae }
$$

Orthotrichum anomalum Hedw. - 4: bark of Fagus

Orthotrichum speciosum Nees -12, 13: bark of Salix

Ulota crispa (Hedw.) Brid. - 4: bark of Fagus, 5: on Picea twigs, 12: bark of Salix

Hedwigiaceae

Hedwigia ciliata (Hedw.) P. Beauv. var. ciliata - 9: on volcanic rocks

$$
\text { Bryaceae }
$$

Bryum capillare Hedw. var. capillare - 13: on limestone rocks Bryum pseudotriquetrum (Hedw.) P. Gaertn. - 2, 9: on rocks Plagiobryum zieri (Hedw.) Lindb. - 13: on limestone rocks

\section{Mniaceae}

Mnium marginatum (Dicks.) P. Beauv. - 12: on limestone rocks

Plagiomnium undulatum (Hedw.) T. J. Kop. - 13: on earth covered rocks

Pohlia nutans (Hedw.) Lindb. - 13: on earth covered rocks

Pohlia wahlenbergii (F. Weber \& D. Mohr.) A. L. Andrews - 13: on limestone rocks

Rhizomnium punctatum (Hedw.) T. J. Kop. - 10: lignicolous; 13: on irrigated rocks

$$
\text { Aulacomniaceae }
$$

Aulacomnium palustre (Hedw.) Schwaegr. - 7: transition bog

\section{Amlystegiaceae}

Amblystegium serpens (Hedw.) Schimp. - 11: on bark

Campylium chrysophyllum (Brid.) Lange - 2: on limestone rocks

Campylium protensum (Brid.) Kindb. - 7: transition bog

Campylium stellatum (Hedw.) Lange \& C.E.O. Jensen - 7: transition bog 
Sanionia uncinata (Hedw.) Loeske - 5: twigs of a dead spruce; 6: on irrigated soil

Hylocomiaceae

Hylocomium splendens (Hedw.) Schimp. - 5, 12, 13: on limestone rocks

Pleurozium schreberi (Willd. ex Brid.) Mitt. - 13: on soil

Rhytidiadelpus triquetrus (Hedw.) Warnst. - 13: on limestone rocks

Rhytidiaceae

Rhytidium rugosum (Hedw.) Kindb. - 1: on soil

Thuidiaceae

Abietinella abietina (Hedw.) M. Fleisch. - 3: on soil

Abietinella abietina (Hedw.) M. Fleisch. var. hystricosa (Mitt.)

Sakurai - 3: on limestone rocks

Brachytheciaceae

Brachythecium rutabulum (Hedw.) Schimp. -12: on limestone rocks

Homalothecium sericeum (Hedw.) Schimp. - 12: on limestone rocks

Eurhynchium angustirete (Broth.) T.J. Kop. - 12: on limestone rocks

Plagiotheciaceae

Plagiothecium denticulatum (Hedw.) Schimp. - 8: raised bog, on litter

Platydictya jungermannioides (Brid.) Crum - 1: on earth covered soil

Hypnaceae

Calliergonella cuspidata (Hedw.) Loeke - 5: on soil

Ctenidium molluscum (Hedw.) Mitt. - 2, 12, 13: on limestone rocks

Hypnum cupressiforme Hedw. - 11: on bark, 13: on limestone rocks

Orthothecium rufescens (Dicks. ex Brid.) Schimp. - 12, 13: on limestone rocks

Ptilium crista-castrensis (Hedw.) De Not. - 13: on limestone rocks

Calliergonaceae

Straminergon stramineum (Dicks. ex Brid.) Hedenas - 6: on irrigated soil, 11: lignicolous 
Warnstorfia exannulata (Schimp.) Loeske - 8: raised bog, on litter

Neckeraceae

Neckera crispa Hedw. - 12: on limestone rock

Neckera complanata (Hedw.) Hüb. - 12, 13: on limestone rocks

Leskeaceae

Leskeella nervosa (Brid.) Loeske - 12: on limestone rocks

\section{DISCUSSION}

Trichocolea tomentella (Ehrh.) Dumort. - it is reported only from two localities from the Bihor Mountains: Galbena Valley, $750 \mathrm{~m}$ alt., 08.1999 (Jakab 2000) and Cetăţile Ponorului, AB 46³3'55"N / 22²2'15"E, $1050 \mathrm{~m}$ alt., 3.09.2007, leg. \& det. Ştefănuţ S. [BUCA B3827]. Based on Mohan (1998) it occurs sporadically in Retezat, Bucegi, Cibin, Arpaşului Mountains, tinovul Poiana Ştampei, Băile Chirui, Valea Ialomiţei, Cascada cu şapte scări, Piatra Mare Mountain, Iezer - Păpuşa Mountains.

Abietinella abietina (Hedw.) M. Fleisch. var. hystricosa (Mitt.) Sakurai - it is known only from one locality in Romania from Pângăraţi valley near Piatra Neamţ (Mihai 1976), but missing from the checklist of Mohan (1998). It was collected by us on the limestone rocks of Scăriţa-Belioara. Another record was published by Pócs (2006) from Scărişoara. It is considered nowadays on molecular basis to be a separate species under the old name of Abietinella hystricosa (Mitt.) Broth.

Barbula crocea (Brid.) F. Weber \& D. Mohr - Europeanmediterranean species and is treated as near threatened (NT) in Romania (Ştefănuţ \& Goia, 2012). It is known from few localities in the Apuseni Mountains: Vl. Seacă, Băița and Şuncuiuş, here collected from Ordâncuşa gorge and Cheile Posăgii gorge (Figure 1, 2). Also known just from few localities in the country: Borsec, Piatra Craiului, Bucegi and Ciucaş Mountains, Cristianul Mare and Piatra Mare Mountain (Mohan 1998). 


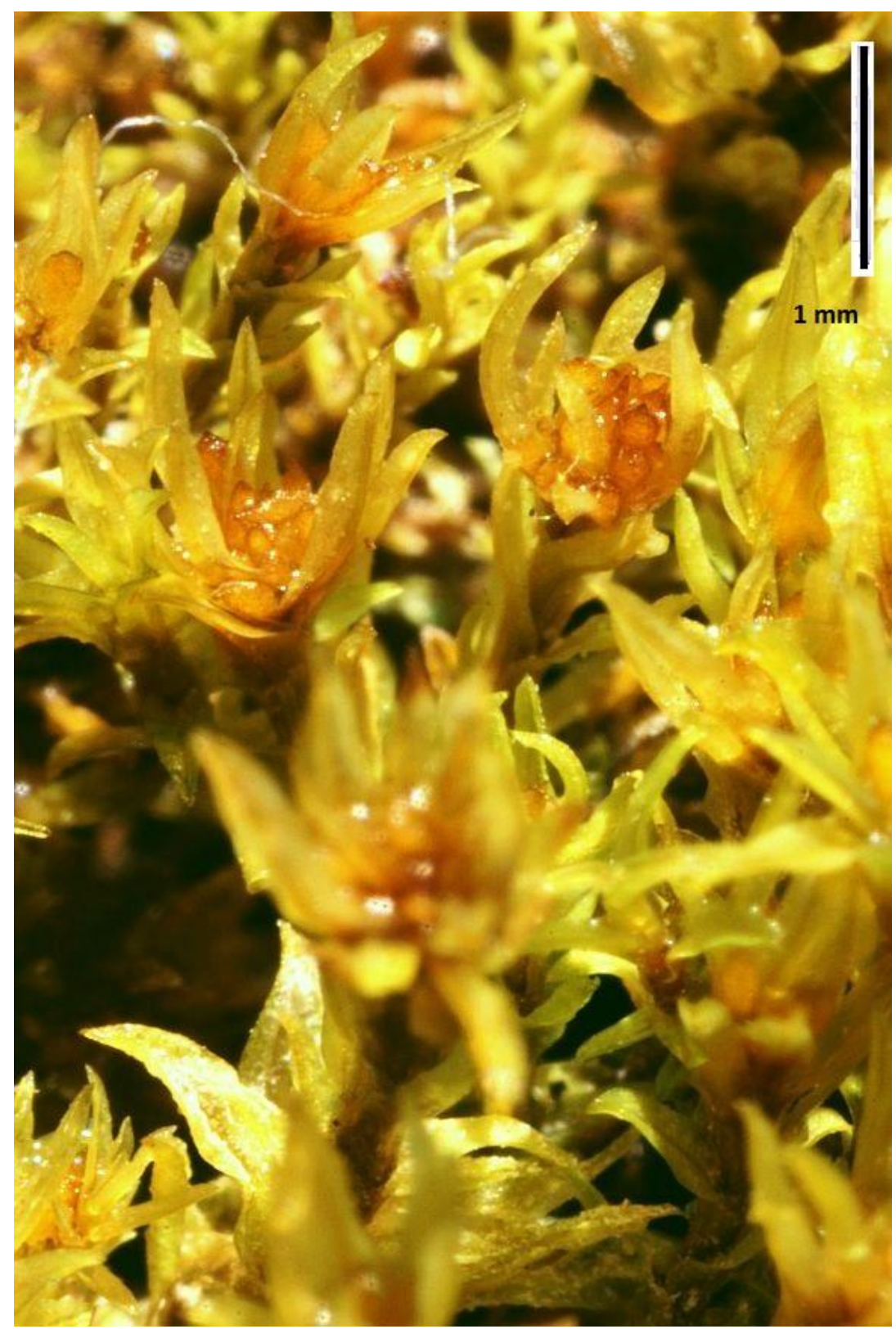

Figure 1. Barbula crocea (Brid.) F. Weber \& D. Mohr, habit (photo: T. Pócs). 


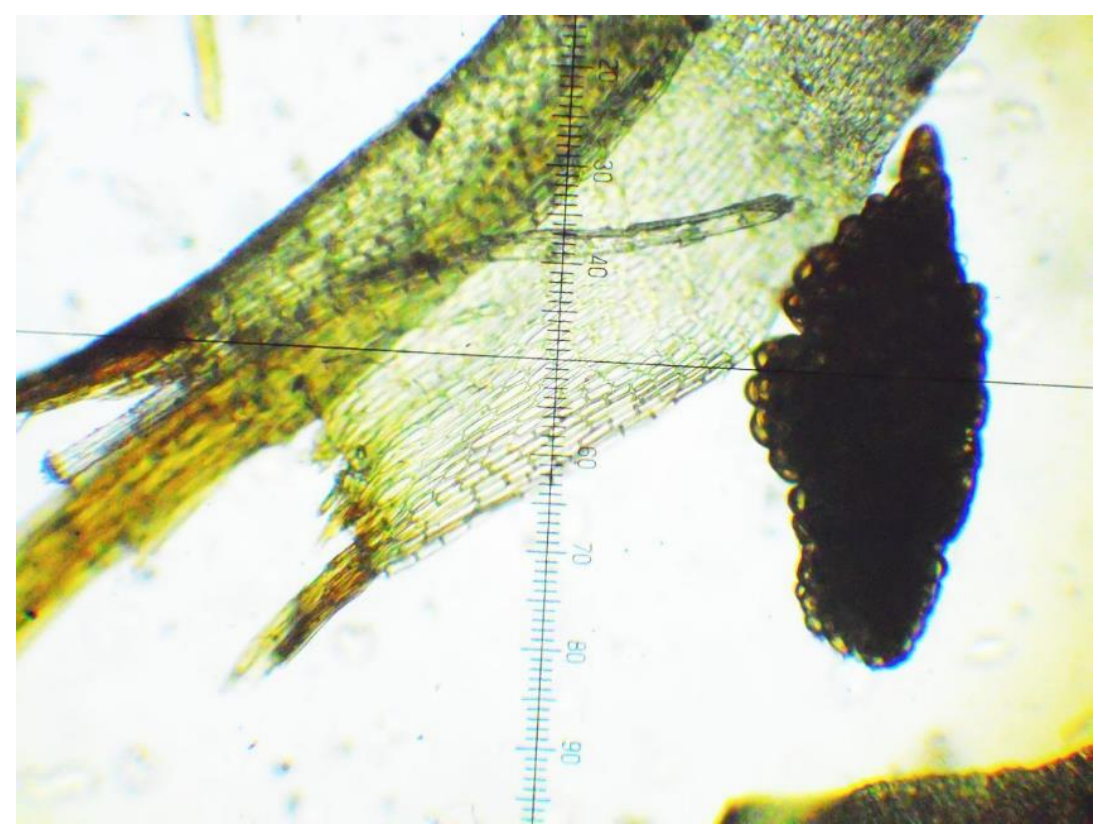

Figure 2. Barbula crocea (Brid.) F. Weber \& D. Mohr, leaf and gemma (scale by 8 $\mu \mathrm{m})$ (photo: T. Pócs).

Seligeria acutifolia (Lindb.) Broth. - is mainly sub-mediterranean and montane taxon (Düll 1984), widely scattered throughout Europe. Besides on Sicily it has also been found on the Italian mainland, in Belgium, (former) Czechoslovakia, France, Germany, Great Britain and Greece, on Ireland, in Norway, Poland, Romania, Sweden and former Yugoslavia (Düll 1985). It occurs in limestone rock crevices, data deficient (DD) in Romania (Ştefănuţ \& Goia 2012). We have found it on a shaded limestone rocks at the narrowest part of Cheile Ordâncușei gorge at $850 \mathrm{~m}$ alt.

Seligeria patula (Lindb.) Broth. - is an Alpine-Carpathian species, it was observed at several other places in the Bihor Mts., as in Ordâncuşa Valley, Galbena gorge, at Barsa ice cave and from Boghii Valley, near the Oşelu waterfall (Pócs 2006). Seligeria patula, similarly to $S$. tristicha, is always embedded in a thin or thick layer of cryptobiotic crust dominated by cyanobacteria.

Campylium protensum (Brid.) Kindb. - is an endangered species in Romania, many bryfloristical works did not separated it from its 
very close relative and more frequent $C$. stellatum. The high red list category of $C$. protensum should be reevaluated after taxonomical revision of herbarium specimens and future fieldworks, to provide the reliable distribution.

Platydictya jungermannioides (Brid.) Crum - the species is treated as near threatened (NT) in Romania (Ştefănuț \& Goia 2012). Distribution based on Mohan (1998): Piatra Craiului Mountains, Cristianul Mare Mountains, Cluj cimitery, Cărpiniş forest, Retezat Mountains, Laptelui Mountains, Puzdra Mountains, Neamţ county: Măgura-Petricica, Bucegi Mountains, Făgăraş Mountains, Ouşoru Mountains.

Syntrichia norvegica F. Weber - this species is treated as vulnerable in Romania (Ştefănuţ \& Goia 2012). New to the Apuseni Mountains communicated in the country only from Retezat, Făgăraş Mountains and from Schitul Obârşia Ialomiţei (Mohan 1998). Circumpolar, Arctic-montane, strongly calcicolous element. Montane and northern Europe north to Svalbard, Iceland, Caucasus, Turkey, Cyprus, Asia, Madeira, N. Africa, N. America, Greenland and Mexico (Smith 2004).

Acknowledgement - The first author thanks to the grant EFOP-3.6.1-16-201600001 "Complex improvement of research capacities and services at the Eszterházy Károly University" for supporting this article. The authors would like to thank our reviewers, Irina Goia (Cluj-Napoca) and Péter Szűcs (Eger) for their useful comments to the manuscript.

\section{REFERENCES}

Boros, Á. (1942a). Bryologische Studien am Rande des Bihargebirges. Scripta Botanica Musei Transsilvanici 1: 31-37.

Boros, Á. (1942b). A Sebes Kőrös menti barlangok szádájának növényvilága. (Die Vegetation der Höhleneingänge längs des Flusses Sebes Kőrös im Bihargebirge.) Scripta Botanica Musei Transsilvanici 1: 152-156.

Boros, Á. (1951). Bryologische Beiträge zur Kenntnis der Flora von Ungarn und der Karpaten. Acta Biologica Academiae Scientiarum Hungaricae 2(1-3): 369-409.

Boros, Á. \& VAJDA, L. (1967). Bryologische Beiträge zur kenntnis der Flora Transilvaniens. Revue Bryologique et Lichénologique 35(1-4): 216-253.

Boros, Á. \& VAJDA, L. (1974). Bryogeographische Forschungen im Karstgebiet des Bihar-Gebirges. Acta Botanica Academiae Scientiarum Hungaricae 20(1-2): 311. 
Csató, J. (1885). A Mluha nevű tó (Teu Mluhi) és viránya. Magyar Növénytani Lapok 9(93): 1-8.

Csû́Rös, I. (1958). Cercetări de vegetaţie pe masivul Scărişoara-Belioara. Studia Universitatis "Babeş-Bolyai" Cluj, Series Biologia 3(7): 105-128.

Csû́Rös, I. (1981). A Nyugati-Szigethegység élővilágáról. Tudományos és Enciklopédiai Könyvkiadó, Bukarest, 303 pp.

DüLL, R. (1984). Distribution of the European and Macaronesian mosses (Bryophytina) I. Bryologische Beiträge 4: 1-110.

DüLL, R. (1985). Distribution of the European and Macaronesian Mosses (Bryophytina) Part II. Bryologische Beiträge 5: 101-232.

Goffinet, B. \& ShaW, A. J. (eds.) (2009). Bryophyte biology. Cambridge University Press, Cambridge, $565 \mathrm{pp}$.

GoiA, I. (2001). Brioflora de pe substrat lemnos din bazinul superior al Arieșului. Studii briotaxonomice, corologice, ecologice şi cenologice. Cluj-Napoca ( $P h D$ Thesis). $312 \mathrm{pp}$.

GoIA, I. (2005). Distribution of some rare bryophytes from Romania identified from the upper basin of the Arieș River (II). Contribuţii Botanice 40: 101-104.

GoiA, I. \& MăTASE, D. (2001). Bryofloristical research in the Someșul Cald gorges. Contribuţii Botanice 36: 15-24.

GoIA, I. \& SCHUMACKER, R. (2000). Researches on the bryophytes from rotten wood in the Arieșul Mic Basin. Contribuții Botanice 1: 15-22.

Goia, I. \& Schumacker, R. (2002). The bryophytes from rotten wood in the Arieşul Mare Basin. Contribuţii Botanice 37: 85-94.

GoiA, I. \& SCHUMACKER, R. (2003a). The study of corticolous bryophyte communities from the Arieșul Mic basin. Contribuţii Botanice 38: 57-68.

GoiA, I. \& SCHUMACKER, R. (2003b). Decaying wood communities from the upper basin of the Arieș River conserving rare and vulnerable bryophytes. Contribuţii Botanice 38: 173-181.

GoiA, I. \& SCHUMACKER, R. (2004). The study of corticolous bryophyte communities from the Arieșul Mare Basin. Contribuţii Botanice 39: 57-68.

GoIA, I. \& ŞTEFĂNUT, S. (2004). Rare bryophytes from Romania, identified from the upper basin of the Arieș river. Contribuţii Botanice 39: 33-36.

GYőRFFY, I. (1903). Bryológiai jegyzetek (Bryologische Notizen). Magyar Botanikai Lapok 2: 146-150.

Hill, M. O., Bell, N., Bruggeman-Nannenga, M. A., Brugués, M., Cano, M. J., Enroth, J., Flatberg, K. I., Frahm, J.-P., Gallego, M.T., Garilleti, R., Guerra, J., Hedenäs, L., Holyoak, D. T., Hyvönen, J., Ignatov, M. S., Lara, F., Mazimpaka, V., MuÑoz, J. \& SöDERSTRÖM, L. (2006). An annotated checklist of the mosses of Europe and Macaronesia. Journal of Bryology 28: 198-267.

https://doi.org/10.1179/174328206x119998

JАКАВ, G. (1999). Three bryophytes new to Romania. Studia Botanica Hungarica. 30-31: 87-94.

JАКАВ, G. (2000). Adatok a Pádis karsztvidékének (Bihari-hegység) mohaflórájához. Crisicum 3: 65-72.

LÜTH, M. (2002). Dicranum transsylvanicum (Musci, Dicranaceae), a new species from Romania. Cryptogamie, Bryologie 23(1): 17-21.

Minal, G. (1976). Briofite noi sau rare in flora Romaniei. Studii si Cercetari de Biologie 28: 23-27. 
MoнAN, G. (1998). Catalogul briofitelor din România. Acta Botanica Horti Bucurestiensis. Ed. Univ. Bucureşti, 432 pp.

Ochyra, R., Zarnowiec, J. \& Bednarek-Ochyra, H. (2003). Census Catalogue of Polish Mosses. Biodiversity of Poland. W. Szafer Institute of Botany, Polish Academy of Sciences, Kraków. Vol. 3, 372 pp.

PÁLL, ş. (1960). Contribuţii la cunoaşterea brioflorei din Cheia Turului, Cheile Turzii, Colţii Trascăului şi Munții Bedeleului. (Contribution à la connaissance de la bryoflore de Cheia Turului, de Cheile Turzii, de Colţii Trascăului et des Monts de Bedeleu.) Studia Universitatis "Babeş-Bolyai", Cluj, Series Biologia 2(2): $1-5$.

PálL, Ş. (1962): Contribuţii la cunoaşterea brioflorei de pe Muntele Pietrele Albe (Masivul Vlădeasa). Studii şi cercetări de biologie - Seria biologie vegetală 14: 30-32.

PálL, Ş. (1963). Contribution à l'etude de la bryoflore des monts Apuseni (Depresion de Padis et Izbucul Ponorului). Studia Universitatis "Babeş-Bolyai", Cluj, Series Biologia 1: 7-12.

Perşoiu, A. \& Pazdur, A. (2011). Ice genesis and its long-term mass balance and dynamics in Scarisoara Ice Cave, Romania. The Cryosphere 5: 45-53. https://doi.org/10.5194/tc-5-45-2011

PÉTERFI M. (1908). Adatok a Biharhegység mohaflórájának ismeretéhez. Mathematikai Természettudományi Közlemények 30: 261-332.

PÉTERFI, M. (1910). Adatok Magyarország mohaflórájához. Beiträge zur Kenntnis der Moosflora Ungarns. Magyar Botanikai Lapok 9(10-12): 1-14.

Plămadă, E. (1998). Flora briologica a Romaniei, Clasa Musci. Vol. I. Fasc. I. Sphagnales - Andreaeales - Tetraphidales - Buxbaumiales - Schistostegales Polytrichales - Fissidentales - Archidiales - Seligeriales. Cluj-Napoca: Presa Universitară Clujană, 230 pp.

PLĂMADĂ, E. \& GOIA, I. (1994). Flora şi vegetaţia briofitică din rezervaţia naturală Cheile Turzii. (The bryophytic flora and vegetation in the natural reservation Turda Canyon.) Contribuţii Botanice 1993-1994: 85-95.

PlăMADĂ, E., GoIA, I. \& Dumitru, C. (2000). New or extremely rare species recorded in the Romanian bryoflora. Contribuţii Botanice 35(1): 23-34.

Pócs, T. (2005). Aerophytic cyanobacteria from the Munţii Apuseni (Romanian Western Carpathians, Transylvania), I. The epilithic crusts at the entrance of Huda lui Papară Cave. Kanitzia 13: 99-108.

Pócs, T. (2006). Adatok az Erdélyi Szigethegység mohaflórájának ismeretéhez. (Contribution to the Bryoflora of Romanian Western Carpathians.) In: KaLAPos, T. (ed.): Jelez a Flóra és a Vegetáció. A 80 éves Simon Tibort köszöntjük. Scientia kiadó, Budapest, pp. 9-24.

Pop, I. \& HodişAn, I. (1967). Aspecte de vegetaţie din Cheile Ordîncuşii. Studia Universitatis Babes-Bolyai, Series Biologia 2: 7-12.

Pop, I., CRISTEA, V. \& HodișAN, I. (2000). Vegetaţia judeţului Cluj (Studiu fitocenologic, ecologic, bioeconomic şi eco-protectiv). Contribuţii Botanice 35(2): 5-254.

RAŢıU O. (ed., colab.) (1966). Flora şi vegetaţia Rezervaţiei Naturale "Defileul Crişului Repede" (Die Flora und Vegetation des Naturschutzgebietes der Crişului Repede Talenge). Contribuţii Botanice 1: 1-272.

Sass-Gyarmati, A., Molnár, K., Orbán, S., Pócs, T. \& Erzberger, P. (2005a). The cryptogamic flora of the Zgurăşti Cave and surroundings (Apuseni Mountains, 
Romania). XVII International Botanical Congress. Vienna, Austria: Austria Center Vienna, 17-23 July 2005 Abstracts, p. 619.

Sass-Gyarmati, A., Pócs, T. \& ORBÁn, S. (2005b). Contribution to the knowledge of the bryoflora of Natural Reserve Detunata (Metaliferi Mountains, Romania). Studia Botanica Hungarica 36: 123-130.

Sass-Gyarmati, A., Goia, I. \& Pócs, T. (2008a). The distribution of Metzgeria violacea in the Apuseni Mountains (Romanian Western Carpathians). Folia Cryptogamica Estonica 44: 121-124.

Sass-Gyarmati, A., Molnár, K., Orbán, S., Pócs, T. \& Erzberger, P. (2008b). The cryptogamic flora of the Zgurăşti Sinkhole System and its surroundings. (Apuseni Mountains, Romania). Kanitzia 16: 25-44.

SASS-GyARmati, A. \& Pócs, T. (2017). Bryofloristical data from the Apuseni Mountains (Romanian Western Carpathians, Transilvania). Acta Biologica Plantarum Agriensis 5(2): 34-51. https://doi.org/10.21406/abpa.2017.5.2.34

Simon, T. (1960). Beitrage zur kenntnis der vegetation des Bihar (Bihor)-Gebirges. Annales Universitatis Scientiarum Budapestinensis (Sectio biologica) 8: 253273.

Smith, A. J. E. (2004). The Moss Flora of Britain and Ireland. Cambridge University Press, Cambridge, 1012. pp. https://doi.org/10.1017/CB09780511541858

Söderström, L., HagborG, A., von Konrat, M., Bartholomew-Began, S., Bell, D., Briscoe, L., Brown, E., Cargill, D. C., Costa, D. P., Crandall-Stotler, B. J., Cooper, E. D., Dauphin, G., Engel, J. J., FeldberG, K., Glenny, D., Gradstein, S. R., He, X., Heinrichs, J., Hentschel, J., Ilkiu-Borges, A. L., Katagiri, T., Konstantinova, N. A., Larraín, J., Long, D. G., Nebel, M., Pócs, T., Puche, F., Reiner-Drehwald, E., Renner, M. A. M., SassGyarmati, A., Schäfer-Verwimp, A., Moragues, J. G. S., Stotler, R. E., Sukkharak, P., Thiers, B. M., Uribe, J., VáŇA, J., Villarreal, J. C., Wigginton, M., Zhang, L. \& Zhu, R.-L. (2016). World checklist of hornworts and liverworts. PhytoKeys 59: 1-828. https://doi.org/10.3897/phytokeys.59.6261

Strug, K., Piasecki, J., Szymanowski, M., Sawiński, T., \& Zelinka, J. (2006). Quantitative characteristics of the bottom ice in the Demänovská Ice Cave (Slovakia). In: Bella, P. (ed.): Výskum, využívanie a ochrana jaskýň, 26.-29.09.2005, Demänovská Dolina, Conference Proceeding: Slovak Caves Administration, Liptovský Mikuláš, pp. 167-174.

ŞTEFĂnuȚ, S. (2008). The Hornwort and Liverwort Atlas of Romania. Edit. Ars Docendi - Universitatea din Bucureşti, Bucureşti, 510 pp.

ŞTEFĂnuȚ, S. \& GoIA, I. (2012). Checklist and Red List of Bryophytes of Romania. Nova Hedwigia 95(1-2): 59-104. https://doi.org/10.1127/0029-5035/2012/0044

ŞTEFurEAC, T. (1975). Consideration sur le caractère bryogeographique des Monts Apuseni (Carpathes Occidentales) de Roumanie. Revue Bryologique et Lichénologique 41(3): 309-314.

ŞTefureAC, T. (1977). Noi contribuţii la ecologia şi corologia Sfagnaceelor din România. Studii şi Comunicari Muz. Șt. Nat. Bacău 1976-1977: 97-112.

(submitted: 01.08.2018, accepted: 16.10.2018) 


\section{APPENDIX}

List of collecting sites from the Apuseni Mts. (Nyugati Szigethegység):

1. Munţii Gilăului (Gyalui Havasok), Rezervaţia Şesu Craiului Scăriţa Belioara (Bélavár). Şesu Craiului ridge, $2.5 \mathrm{~km} \mathrm{~N}$ of Poşaga de Sus (Felsőpodsága). 1350-1370 m alt. N 46²9’46.1", E $23^{\circ} 21^{\prime} 56.9^{\prime \prime}$ Hab.: Dry Festuco-Caricetum humilis sward on the steep $\left(45^{\circ}\right) \mathrm{S}$ slope above the limestone cliffs. Date: 22. Oct. 2006 Coll.: T. Pócs, I. Goia \& Z. Tóth. No. 06090

2. Munţii Gilăului (Gyalui Havasok), Cheile Poşegii, $500 \mathrm{~m}, \mathrm{~N}$ of Mănăstirea Poşaga in the gorge. N 46²7'30.5", E 23²3'53.1" Hab.: N facing, shady limestone rocks with scattered Sesleria rigida and abundant Selaginella helvetica. Date: 23. Oct. 2006 Coll.: T. Pócs \& Z. Tóth. No. 06093

3. Munţii Gilăului (Gyalui Havasok), Rezervaţia Şesu Craiului Scăriţa Belioara (Bélavár). Belioara Valley below the huge, SSE facing cliffs at 710-720 m alt. N 46²9.143', E 2322.229' Hab.: Limestone scree covered by Corylus bush. Date: 23. Oct. 2006. Coll.: T. Pócs \& Z. Tóth. No. 06095

4. Munţii Gilăului (Gyalui Havasok), Rezervat Şesu Craiului - Scăriţa Belioara (Bélavár). Belioara Valley below the huge, SSE facing cliffs at 920-1000 m alt. N 46²9.502', E 2322.954'. Hab.: Beech (Fagus silvatica) forest. Date: 23. Oct. 2006. Coll.: T. Pócs \& Z. Tóth. No. 06096

5. Munţii Gilăului (Gyalui Havasok), complex of Muntele Mare (Öreghavas). Valea Mare NE of Bistra below Cheleteni village, at 920 m alt. N 46²6'40", E $23^{\circ} 07^{\prime} 44.5^{\prime \prime}$. Hab.: Acidiphilous spruce forest on volcanic rocks and scree. Date: 24. Oct. 2006. Coll.: T. Pócs \& Z. Tóth. No. 06097

6. Munţii Gilăului (Gyalui Havasok). W end of the plateau of Muntele Mare (Öreghavas) near the saddle to Vf. Prislop, at $1685 \mathrm{~m}$ alt. $\mathrm{N}$ 462' $29^{\prime} 30.9^{\prime \prime}$, E $23^{\circ} 12^{\prime} 31.4^{\prime \prime}$. Hab.: Complex of transition, spring and raised peat bog. Date: 24. Oct. 2006. Coll.: T. Pócs \& Z. Tóth. No. 06098

7. Munţii Gilăului (Gyalui Havasok). NW part of the plateau of Muntele Mare (Öreghavas), W from the mean summit, at 17151725 m alt. N 46²9'36.4", E 2312'50.2". Hab.: Transition spring bog with Carex hartmanii. Date: 24. Oct. 2006. Coll.: T. Pócs \& Z. Tóth. No. 06099 
8. Munţii Gilăului (Gyalui Havasok). NW part of the plateau of Muntele Mare (Öreghavas), $1.5 \mathrm{~km} \mathrm{~W}$ from the mean summit, near the springs of Iara River, place called "Gemenele Ţiganului" at 1716-1725 $\mathrm{m}$ alt. N 46²9'51.7", E 2313'09.0". Hab.: Big, raised bog (Eriophoro vaginatae- Sphagnetum). Date: 24. Oct. 2006. Coll.: T. Pócs \& Z. Tóth. No. 06100

9. Munţii Gilăului (Gyalui Havasok), complex of Muntele Mare (Öreghavas). Valea Mare NE of Bistra at 716 m alt. N 46²4'37.5", E $23^{\circ} 06^{\prime} 34.1^{\prime \prime}$. Hab.: Spruce forest on volcanic ground with open lava rocks. Date: 24. Oct. 2006. Coll.: T. Pócs \& Z. Tóth. No. 06101

10. Munţii Bihorului (Bihar Hegység), S ridge of Dealu Bocului $1 \mathrm{~km}$ NW of Scărişoara Ice Cave, at 1200-1290 m alt. N 46²9'39.9", E 22048'19.3". Hab.: Mixed Fagus-Abies-Picea forest on limestone ground, alternating with openings. Date: 25. Oct. 2006. Coll.: T. Pócs \& Z. Tóth. No. 06102

11. Munţii Bihorului (Bihar Hegység), 200-700 m N of Scărişoara Ice Cave near the Avenul din Şesuri sinkhole, at $1200 \mathrm{~m}$ alt. $\mathrm{N}$ 46²9'27.6", E 2248'22.7". Hab.: Mixed Fagus-Abies-Picea forest on limestone ground. Date: 25. Oct. 2006. Coll.: T. Pócs \& Z. Tóth. No. 06103

12. Munţii Bihorului (Bihar Hegység), Cheile Ordâncuşei gorge NE of Gârda de Sus village, near the Poarta lui Ionele cave, at $775 \mathrm{~m}$ alt. N 46²7'59.8", E 2250'17.55". Hab.: Shady limestone cliffs with Seslerietum rigidae. Date: 25. Oct. 2006. Coll.: T. Pócs \& Z. Tóth. No. 06104

13. Munţii Bihorului (Bihar Hegység), northern, very narrow part of Cheile Ordâncuşei gorge ("Seligeria Canyon") NE of Gârda de Sus

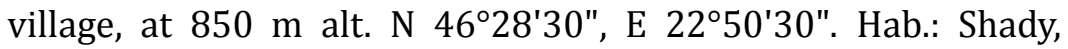
vertical limestone cliffs, almost no phanerogams. Date: 25 . Oct. 2006. Coll.: T. Pócs \& Z. Tóth. No. 06105 